\title{
Lorentz TEM Image Simulations of Dzyaloshinskii Domain Walls Under an In- Plane Magnetic Field
}

\author{
Maxwell P. Li $^{1}$, Marc De Graef ${ }^{1}$ and Vincent Sokalski ${ }^{1}$ \\ ${ }^{1 .}$ Dept. of Materials Science and Engineering, Carnegie Mellon Univ., Pittsburgh, PA 15213, USA
}

Topologically protected magnetic structures stabilized by the Dzyaloshinskii-Moriya Interaction (DMI), such as skyrmions and chiral domain walls (DWs), have garnered a great deal of attention in recent years due to the unprecedented efficiency with which they can be manipulated by electric current. The DMI energy, $E=-\boldsymbol{D} \cdot\left(\boldsymbol{S}_{\mathbf{1}} \times \boldsymbol{S}_{\mathbf{2}}\right)$, favors canting of neighboring magnetic moments and can only have a net effect in systems without inversion symmetry. This is found in bulk non-centrosymmetric magnetic structures like $\mathrm{FeCoSi}_{2}$ [1], but more recently has been engineered in perpendicularly magnetized thin films with an asymmetric stacking sequence [2]. The result is an effective in-plane magnetic field, $\mathrm{H}_{\mathrm{DMI}}$, that acts perpendicular to any existing DWs, thereby stabilizing the Néel configuration with preferred chirality over the magnetostatically favored Bloch type.

Existing methods to measure $\mathrm{H}_{\mathrm{DMI}}$ include Brillouin light scattering [3] or domain expansion under the influence of an in-plane magnetic field [4]. Although such techniques provide valuable insight, they are inherently indirect and significant variation has been observed among them [5]. As a path to directly probe interfacial DMI, we present Fresnel mode Lorentz TEM image calculations of Dzyaloshinskii DWs subjected to an in-plane magnetic field, $\mathrm{H}_{\mathrm{x}}$. We simulate the evolving internal structure of the wall through micromagnetic energy minimization using $\operatorname{Mumax}^{3}$ [6]. The magnetic properties were fixed at $\mathrm{A}=1 \times 10^{-11} \mathrm{~J} / \mathrm{m}, M_{\mathrm{s}}=600 \mathrm{kA} / \mathrm{m}, \mathrm{K}_{\text {eff }}=3 \times 10^{5} \mathrm{~J} / \mathrm{m}^{3}$, and $\mu_{\mathrm{o}} \mathrm{H}_{\mathrm{DMI}}=0.1 \mathrm{~T}$ for DWs and $\mathrm{A}=1 \times 10^{-11} \mathrm{~J} / \mathrm{m}$, $\mathrm{M}_{\mathrm{s}}=600 \mathrm{kA} / \mathrm{m}, \mathrm{K}_{\mathrm{eff}}=2.5 \times 10^{5} \mathrm{~J} / \mathrm{m}^{3}$, and $\mu_{\mathrm{o}} \mathrm{H}_{\mathrm{DMI}}=0.150 \mathrm{~T}$ for labyrinth configurations.

Simple two domain configurations separated by a planar DW with variable Bloch and Néel component are shown in Fig. 1 with increasing $\mathrm{H}_{\mathrm{x}}$. Under the calculated Fresnel imaging configuration, only the Bloch component deflects the electron beam perpendicular to the DW, which is necessary to observe any contrast. As $\mathrm{H}_{\mathrm{DMI}}$ is typically much larger than what is required to stabilize a pure Néel wall, revealing the internal structure through sample tilting alone can not be used to directly determine $\mathrm{H}_{\mathrm{DMI}}$. Instead, through application of an in-plane field, $\mathrm{H}_{\mathrm{x}}$, anti-parallel to $\mathrm{H}_{\mathrm{DMI}}$ it is possible to identify the point at which Fresnel contrast is a maximum, which will occur when $\mathrm{H}_{\mathrm{x}}$ cancels $\mathrm{H}_{\mathrm{DMI}}$ and the wall becomes fully Bloch type. Conversely, application of a field along the Néel DW will initially give enhanced contrast associated with rotation of the internal magnetization towards Bloch type. The impact of $\mathrm{H}_{\mathrm{x}}$ on Lorentz images is shown in Fig 1c where contrast between adjacent pixels is calculated by $|\Delta I| / \sum \boldsymbol{I}$.

Although either technique described above is appropriate for extracting $\mathrm{H}_{\mathrm{DMI}}$, real magnetic thin films will often relax to a labyrinth configuration comprising a distribution of DW orientations with respect to the applied field. We have simulated the response of such configurations to serve as a comparison with future experimental Lorentz TEM images (Fig. 2). In addition to the evolving Bloch-Néel components, vertical Bloch lines emerge, which show strikingly significant contrast relative to that associated with the wall. These topological defects are singularities in the internal magnetization, which are themselves stabilized by the interfacial DMI [7]. We observe that these defects drift towards extrema in the wall profile under the applied field before annihilating at some critical field, which is governed by the 
magnitude of DMI. Bloch lines are believed to greatly impact DW properties, but have been difficult to study experimentally due to the requirement for Lorentz TEM and in-situ application of a planar magnetic field.

The models presented here will ultimately serve as a reference for experimental images taken on a FEI Titan TEM with a sample mount that allows for application of an in-situ in-plane field. This will offer a direct method for measuring interfacial DMI and evaluating the properties of related topological defects.
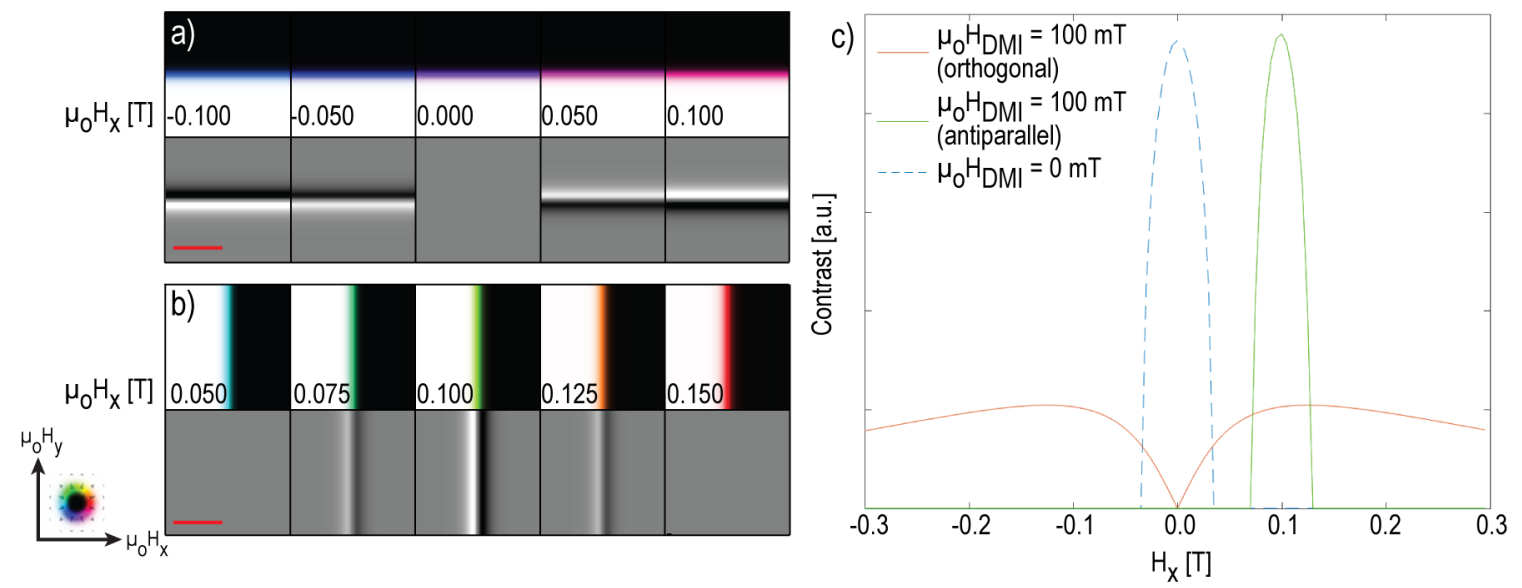

Figure 1. Micromagnetic and Lorentz TEM simulations of a Dzyaloshinskii DW subject to an in-plane field with a) $\mathrm{H}_{\mathrm{x}}$ orthogonal to $\mathrm{H}_{\mathrm{DMI}}$ and b) $\mathrm{H}_{\mathrm{x}}$ antiparallel to $\mu_{\mathrm{o}} \mathrm{H}_{\mathrm{DMI}}=100 \mathrm{mT}$. Scale bar is $50 \mathrm{~nm}$. c) Plot depicting Lorentz TEM image contrast at domain wall with respect to applied field, $\mathrm{H}_{\mathrm{x}}$.

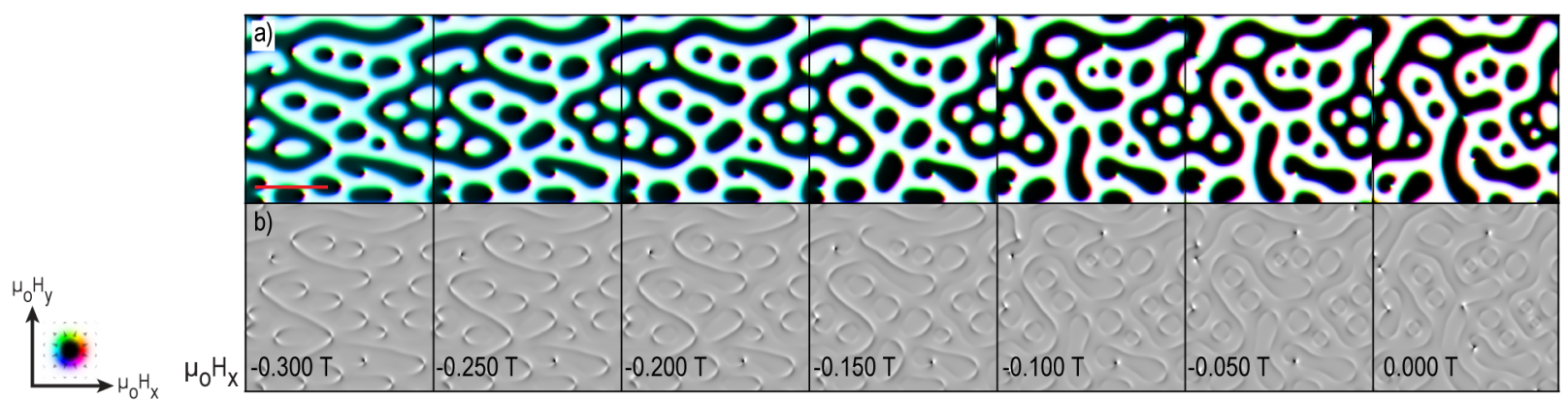

Figure 2. Micromagnetic simulations (a) and simulated Fresnel LTEM images (b) of labyrinth domain structures subjected to an in-plane magnetic field with $\mu_{0} \mathrm{H}_{\mathrm{DMI}}=150 \mathrm{mT}$. Scale bar is $200 \mathrm{~nm}$.

[1] X. Z. Yu, et al., Nature 465 (2010).

[2] A. Thiaville, et al., Epl 100 (2012).

[3] K. Di, et al., Physical Review Letters 114 (2015).

[4] S.-G. Je, et al., Physical Review B 88 (2013).

[5] R. Soucaille, et al., Physical Review B 94 (2016).

[6] A. Vansteenkiste, et al., Aip Advances 4 (2014).

[7] Y. Yoshimura, et al., Nature Physics 12 (2016). 